Pacific Journal of Mathematic 


\section{ON ABELIAN PSEUDO LATTICE ORDERED GROUPS}

\section{J. ROGER TELleR}

Throughout this paper po-group will mean partially ordered abelian group. A subgroup $H$ of a po-group $G$ is an $o$-ideal if $H$ is a convex, directed subgroup of $G$. A subgroup $M$ of $G$ is a value of $0 \neq g \in G$ if $M$ is an $o$-ideal of $G$ that is maximal without $g$. Let $\mathscr{C}(g)=\{M \cong G \mid M$ is a value of $g\}$ and $\mathscr{C} *(g)=\bigcap \mathscr{C}(g)$. Two positive elements $a, b \in G$ are pseudo disjoint ( $p$-disjoint) if $a \in \mathscr{C}^{*}(b)$ and $b \in \mathscr{C l}^{*}(a)$, and $G$ is a pseudo-lattice ordered group ( $p l$-group) if each $g \in G$ can be written $g=a-b$ where $a$ and $b$ are $p$-disjoint.

The main result of $\S 2$ shows that every $p l$-group $G$ is a Riesz group. That is, $G$ is semiclosed $(n g \geqq 0$ implies $g \geqq 0$ for all $g \in G$ and all positive integers $n$ ), and $G$ satisfies the Riesz interpolation property; if, whenever $x_{1}, \cdots, x_{m}, y_{1}, \cdots, y_{n}$ are elements of $G$ and $x_{i} \leqq y_{j}$ for $1 \leqq i \leqq m, 1 \leqq j \leqq n$, then there is an element $z \in G$ such that $x_{i} \leqq z \leqq y_{j}$.

In $\S 3$, we determine which Riesz groups are also pl-groups. In the final section it is shown that each pair of $p$-disjoint elements $a, b$ determines an $o$-ideal $H(a, b)$ with the property that if $a-b=x-y$ where $x$ and $y$ are also $p$-disjoint, then $H(a, b)=H(x, y)$ and $a-x=$ $b-y \in H(a, b)$.

The concept of a pl-group has been introduced by Conrad [1]. For each $g \in G, \mathscr{C}^{*}(g)$ exists by definition, and in particular, $\mathscr{K}^{*}(0)=G$. In $\S 2$ we list a number of properties of $p l$-groups that will be used. We adopt the notation $a \| b$ for $a \geqq b$ and $b \geqq a$. If $S$ is a subset of a po-group $G$ and $a \in G$, the notation $a>S$ means $a>s$ for all $s \in S$. If $H$ is an $o$-ideal of a po-group $G$, a natural order is defined in $G / H$ by setting $X \in G / H$ positive if $X$ contains a positive element of $G$. All quotient structures will be ordered in this manner. Finally, $G^{+}=\{x \in G \mid x \geqq 0\}$.

2. Some properties of $p l$-groups. We first list a number of properties of $p l$-groups. The proofs of these may be found in [1]. If $G$ is a $p l$-group, then

(1) $G$ is semiclosed.

(2) $G$ is directed.

(3) The intersection of $o$-ideals of $G$ is an $o$-ideal.

(4) If $g \in G$ and $M \in \mathscr{M}(g)$ and $M^{\prime}$ is the intersection of all $o$-ideals of $G$ that properly contain $M$, then $g \in M^{\prime}, M^{\prime} / M$ is 0 -isomorphic to a naturally ordered subgroup of the real numbers and, if $M<X \in G / M \backslash M^{\prime} / M$, then $X>M^{\prime} / M$. 
(5) If $K$ is an $o$-ideal of $G$, then $K$ and $G / K$ are $p l$-groups.

(6) If $K$ is an $o$-ideal of $G$ and $g \in G \backslash K$, then there is $M \in \mathscr{C}(g)$ such that $M \supseteqq K$.

(7) If $g=a-b$ where $a$ and $b$ are $p$-disjoint, then $\mathscr{l l}(g)=$ $\mathscr{L C}(a) \cup \mathscr{L C}(b)$.

(8) A nonzero element $g \in G$ is positive if and only if $g+M>M$ for all $M \in \mathscr{L}(g)$.

(9) If $a$ and $b$ are $p$-disjoint and $g \leqq a, g \leqq b$, then $n g \leqq a$ and $n g \leqq b$ for all $n>0$.

(10) If $a$ and $b$ are $p$-disjoint, then no value of $a$ is comparable to a value of $b$.

The following set of propositions leads to the first theorem which states that every $p l$-group is a Riesz group.

(2.1) Let $G$ be a po-group and $g \in G$. If $g=a-b$ where $a$ and $b$ are $p$-disjoint and $z \in G^{+}$such that $z \geqq g$, then each value of $a$ is contained in a value of $z$, and if $a \geqq z$, then $z$ and $z-g$ are $p$-disjoint.

Proof. Let $M \in \mathscr{C}(a)$, then $b \in M$ and $z \geqq g=a-b$ implies $z+b \geqq a \geqq 0$. Hence, $z \notin M$ and there is $M^{\prime} \in \mathscr{M}(z)$ such that $M^{\prime} \supseteqq M$.

From $a \geqq z \geqq 0$ it follows that if $M \in \mathscr{M}(z)$, then $a \notin M$. By the above, $M \in \mathscr{C}(a)$ so $b \in M$. Now $a \geqq z \geqq g$ implies $a-g=b \geqq z-g \geqq 0$ so $z-g \in M$. Similarly, if $M \in \mathscr{M}(z-g)$, then $b \notin M$ so $M \in \mathscr{C}(b)$, $a \in M$ and hence, $z \in M$. Thus, $z$ and $z-g$ are $p$-disjoint.

(2.2) If $G$ is a po-group and $g=a-b=x-y$ where $a$ and $b$ are $p$-disjoint and $x$ and $y$ are positive, then for each

$$
M \in \mathscr{C}(a)[M \in \mathscr{C}(b)]
$$

there is $M^{\prime} \in \mathscr{C}(x)\left[M^{\prime} \in \mathscr{C}(y)\right]$ such that $M^{\prime} \supseteqq M$. In particular, if $x$ and $y$ are $p$-disjoint, $\mathscr{C}(a)=\mathscr{C}(x), \mathscr{L}(b)=\mathscr{C}(y)$ and $a-x=$ $b-y \in \mathscr{L}^{*}(g)$.

Proof. Let $g \in G$ and $g=a-b=x-y$ where $a$ and $b$ are $p$ disjoint and $x$ and $y$ are positive. Since $y \geqq 0$, we have $x \geqq g$ so for $M \in \mathscr{C}(a)$ there is, by (2.1), $M^{\prime} \in \mathscr{C}(x)$ such that $M^{\prime} \supseteqq M$. Similarly for $M \in \mathscr{M}(b)$. If $x$ and $y$ are also $p$-disjoint then, by interchanging the roles of $a$ and $x, y$ and $b$ we obtain $\mathscr{M}(a)=\mathscr{C}(x)$ and $\mathscr{M}(b)=$ $\mathscr{M}(y)$. Thus, $b, y \in \mathscr{C}^{*}(a)$ and $a, x \in \mathscr{C}^{*}(b)$ so

$$
a-x=b-y \in \mathscr{L}^{*}(a) \cap \mathscr{L}^{*}(b)
$$

which is equal to $\mathscr{C}^{*}(g)$ by property (7). 
(2.3) Suppose $G$ is a $p l$-group, $g \in G, g=a-b$ where $a$ and $b$ are $p$-disjoint and $z \in G^{+}$such that $z \geqq g$. If $M \in \mathscr{M}(a-z)$, then either $M \in \mathscr{L}(z)$ and $z+M>a+M$ or $M$ is properly contained in a value of $a$.

Proof. If $M \in \mathscr{M}(a-z)$, then by (4),

$$
a+M>z+M \text { or } a+M<z+M \text {. }
$$

For $M \in \mathscr{L}(z)$ and $M \in \mathscr{C}(a)$, it follows that $z+M>M$ and, from (2.1), that $a \in M$. Hence, $z+M>M=a+M$. For $M \in \mathscr{M}(z)$ and $M \in \mathscr{C l}(a)$, we have $a+M=g+M \leqq z+M$ so $a+M<z+M$. Now if $M \notin \mathscr{C}(z)$, then $a \notin M$ so there is $M^{\prime} \in \mathscr{C}(a)$ such that $M^{\prime} \supseteqq M$. If $M^{\prime}=M$, then $M$ is properly contained in $M^{\prime \prime} \in \mathscr{M}(z)$ so $a$ and $a-z$ are in $M^{\prime \prime}$ and $z \in M^{\prime \prime}$, a contradiction. Thus $M^{\prime}$ properly contains $M$.

LEMma 2.1. If $G$ is a pl-group, $g \in G$ and $z \in G^{+}$such that $z \geqq g$, then there is $x \in G^{+}$such that $z \geqq x$ and $x, x-g$ are p-disjoint. Moreover, if $g=a-b$, with $a$ and $b$ p-disjoint, then there exists such an $x$ with $a \geqq x$.

Proof. Let $G$ be a $p l$-group and $g \in G$. Then $g=a-b$ where $a$ and $b$ are $p$-disjoint. If $z \in G^{+}$and $g \leqq z$, take $x=a$ if $z \geqq a$; and take $x=z$ if $z<a$. The result follows from (2.1).

If $z-a \| 0$, then $z-a=p-q$ where $p$ and $q$ are $p$-disjoint. We first show $\mathscr{C}(q)=\{M \in \mathscr{C}(z-a) \mid z+M<a+M\}$. Let $M \in \mathscr{C}(q)$, then $M \in \mathscr{C}(z-a)$ and $(z-a)+M=-q+M<M$ so $z+M<a+M$. Conversely, if $M \in \mathscr{C}(z-a)$ and $z+M<a+M$, then $M \in \mathscr{L}(p)$ or $M \in \mathscr{C}(q)$. If $M \in \mathcal{L}(p)$, then $q \in M$ so $(z-a)+M=p+M>M$. This implies $z+M>a+M$, a contradiction. Thus, $M \in \mathscr{C}(q)$.

Now let $x=a-q=z-p$, then $x<a$ and $x<z$. If $M \in \mathscr{C}(x)$, then $q \in M$. For if $q \notin M$, then $M \cong M^{\prime} \in \mathscr{C}(q), M^{\prime} \in \mathscr{C}(z-a)$ and $z+M^{\prime}<\alpha+M^{\prime}$. By (2.3), $M^{\prime}$ is properly contained in $M^{\prime \prime} \in \mathscr{C}(\alpha)$. Thus, $x \in M^{\prime \prime}, q \in M^{\prime \prime}$ so $a \in M^{\prime \prime}$ a contradiction. Therefore, $q \in M$ and hence $a \notin M$. We now have $M \neq a+M=x+q+M$ so $M<a+M=$ $x+M$ for all $M \in \mathscr{C}(x)$. By (8), $x \geqq 0$.

To complete the proof we need only show $x \geqq g$, for then the result follows by (2.1). To accomplish this we show $(b-q)+M>M$ for all $M \in \mathscr{C}(b-q)$. Thus, let $M \in \mathscr{C l}(b-q)$. If $M \in \mathscr{C}(q)$, then $M \in \mathscr{l}(z-a)$ and $z+M<a+M$, so $b \notin M$. By (2.3) and (10) there must exist $M^{\prime} \in \mathscr{C}(b)$ such that $M^{\prime}$ properly contains $M$. But $M^{\prime}$ properly containing $M$ implies $b-q, q$ and hence $b \in M^{\prime}$, a contradiction. Thus, $M \notin \mathscr{C}(q)$.

Now since $b \notin M$, there is $M^{\prime \prime} \in \mathscr{C}(b)$ such that $M^{\prime \prime} \supseteqq M$. If 
$M^{\prime \prime} \neq M$, then $b-q \in M^{\prime \prime}$ so $M^{\prime \prime}<b+M^{\prime \prime}=q+M^{\prime \prime}$ and $q \notin M^{\prime \prime}$. By (2.3), every value of $q$ is contained in a value of $a$ so $M^{\prime \prime}$ is contained in a value of $a$, a contradiction. Thus $M^{\prime \prime}=M \in \mathscr{M}(b)$, and as above, it follows that $q \in M$. Consequently, $b-q+M=b+M>M$ so by (8), $b>q$ and $x>g$. This completes the proof.

With Lemma 2.1 we are now able to prove the following.

\section{THEOREM 2.1. Every pl-group is a Riesz group.}

Proof. Since by (1), a pl-group is semiclosed, we need only show a $p l$-group $G$ satisfies the Riesz interpolation property. Without loss of generality, we may assume, $g, u, z \in G$ and $u \geqq 0, z \geqq 0, u \geqq g, z \geqq g$. There exists, by Lemma 2.1 , an element $a \in G^{+}$such that $u \geqq a$ with $a, a-g p$-disjoint. Also, there is $x \in G^{+}$such that $a \geqq x, z \geqq x$ with $x, x-g$-disjoint. Hence, $u \geqq x \geqq 0, z \geqq x \geqq g$ and $G$ is a Riesz group.

We note that the above theorem and Theorem 4.8 in [1] answer affirmitively the open question posed at the end of [2].

3. Sufficient conditions for pseudo-lattice ordering. As a consequence of $\S 2$ we have that every $p l$-group $G$ is a Riesz group that satisfies

$\left(^{*}\right)$ for each $g \in G$, there is $a \in G^{+}$such that $g \leqq a$ and whenever $0 \leqq x, g \leqq x$ then $a \leqq x+h$ for some $h \in \mathscr{C l}^{*}(a) \cap \mathscr{C}^{*}(a-g)$.

To see this let $g \in G$, then $g$ can be written $g=a-b$ where $a$ and $b$ are $p$-disjoint, so $a \in G^{+}$and $g \leqq a$. If $x \in G^{+}$and $x \geqq g$, then, since $G$ is a Riesz group, there is $u \in G$ such that $a \geqq u \geqq 0$ and $x \geqq u \geqq g$. By (2.1), $u$ and $u-g$ are $p$-disjoint and by (2.2) and (7), $a-u \in \mathscr{M}^{*}(a) \cap \mathscr{l}^{*}(a-g)$. By setting $a-u=h$ we have $u=$ $a-h$ so $x \geqq u=a-h$ which implies $x+h \geqq a$.

In this section we show that every Riesz group that satisfies (*) is a pl-group. For the remainder of this section we assume $G$ is a Riesz group that satisfies $\left({ }^{*}\right)$.

LEMMA 3.1. The intersection of o-ideals of $G$ is again an o-ideal.

Proof. Let $M_{\alpha}, \alpha \in J$ be $o$-ideals of $G$ and $M=\bigcap_{\alpha \in J} M_{\alpha}$. Clearly, $M$ is a convex subgroup of $G$. To show $M$ is directed let $g \in M$. By $\left.{ }^{*}\right)$ there is $a \in G$ such that $0 \leqq a, g \leqq a$. Now for each $\alpha \in J, M_{\alpha}$ is directed so $M_{\alpha}$ is a Riesz group. Thus, there are elements $y_{\alpha} \in M_{\alpha}$, $x_{\alpha} \in G$ such that $y_{\alpha} \geqq 0, y_{\alpha} \geqq g, a \geqq x_{\alpha} \geqq g$ and $y_{\alpha} \geqq x_{\alpha} \geqq 0$. Thus, $x_{\alpha} \in M_{\alpha}$ and $a \leqq x_{\alpha}+h_{\alpha}$ for some $h_{\alpha} \in \mathscr{M}^{*}(a) \cap \mathscr{C}^{*}(a-g)$.

Now $x_{\alpha} \in M_{\alpha}$ and $x_{\alpha}+h_{\alpha} \geqq a \geqq x_{\alpha}$ implies $a-x_{\alpha} \in \mathscr{C}^{*}(a)$. Thus, if $a \notin M_{\alpha}$ then there is $M^{\prime} \in \mathscr{L}(a)$ such that $M^{\prime} \supseteqq M_{\alpha}$. But then $x_{\alpha}$, 
$a-x_{\alpha}$ and hence $a \in M^{\prime}$, a contradiction. Thus $a \in M_{\alpha}$ for all $\alpha, M$ is directed and $M$ is an $o$-ideal of $G$.

We note that in the above we have proved that if $a$ satisfies (*) for $g$ and $a \geqq x \geqq 0, x \geqq g$ then $a-x \in \mathscr{L}^{*}(a) \cap \mathscr{C}^{*}(a-g)$.

Lemma 3.2. If $M$ is an o-ideal of $G$, then $M$ and $G / M$ are Riesz groups satisfying $\left({ }^{*}\right)$.

Proof. If $M$ is an $o$-ideal of $G$, then $M$ and $G / M$ are Riesz groups by [2, p. 1393]. If $g \in M$, then let $a \in G$ such that $a$ satisfies (*) for $g$. There then are elements $m \in M^{+}$and $x \in G$ such that $m \geqq g$, $a \geqq x \geqq g$ and $m \geqq x \geqq 0$, which implies $x \in M$ and $a-x \in \mathscr{C}^{*}(a)$. As a consequence of this latter part, $a \in M$. Now if $0 \leqq y \in M$ and $g \leqq y$ then there is $u \in M$ such that $y \geqq u \geqq 0, a \geqq u \geqq g$. Thus, by the remark preceding this lemma, $u=a+h$ where

$$
h \in \mathbb{l}^{*}(\alpha) \cap \mathscr{C l}^{*}(\alpha-g)
$$

and hence $u-a=h \in M$. By Lemma 3.1, every 0 -ideal $M^{\prime}$ of $M$ that is maximal without $a[a-g]$ can be written $M^{\prime}=M \cap \bar{M}$ where $\bar{M} \in \mathscr{C}(a)[\bar{M} \in \mathscr{M}(a-g)]$. Thus, it follows that $h$ belongs to every value of $a$ and every value of $a-g$ in $M$ and $M$ satisfies $\left(^{*}\right)$.

Now let $g+M \in G / M$, and let $a \in G$ such that $a$ satisfies (*) for g. Then $a+M \geqq M$ and $a+M \geqq g+M$. If $M \leqq x+M \in G / M$ and $x+M \geqq g+M$, then there are elements $m_{1}, m_{2} \in M$ such that $x+m_{1} \geqq 0$ and $x+m_{2} \geqq g$. Since $M$ is directed, there is $m \in M$ such that $m \geqq m_{1}, m \geqq m_{2}$.

$\mathrm{By}\left({ }^{*}\right), a \leqq(x+m)+h$ so $a+M \leqq(x+M)+(h+M)$ where

$$
h \in \mathscr{L}^{*}(a) \cap \mathscr{L}^{*}(a-g) \text {. }
$$

Now let $X$ be a value of $a+M$ in $G / M$. Then $X=M^{\prime} / M$ where $M^{\prime}$ is an $o$-ideal of $G$ and $a \in M^{\prime}$. It follows that $M^{\prime} \in \mathscr{M}(a)$ so $h \in M^{\prime}$ and $h+M \in X$. In a similar manner, $h+M$ belongs to every value of $(a-g)+M$ in $G / M$. The proof is complete.

LEMma 3.3. Let $H$ be the intersection of all nonzero o-ideals of G. If $x \in H^{+}, g \in G \backslash H$ and $g<x$, then $g<0$.

Proof. Suppose $H$ is the intersection of all nonzero o-ideals of $G$. If $x \in H^{\dagger}, g \in G \backslash H$ and $g<x$, then $a \leqq x+h$ where $a$ satisfies $\left({ }^{*}\right)$ for $g$ and $h \in \mathscr{C}^{*}(a) \cap \mathscr{C}^{*}(a-g)$. If $a \neq 0$ and $M \in \mathscr{C}(a)$, then $M \neq 0$ so $H \leqq M$ and $x+h \in M$. This implies $a \in M$ since $0 \leqq a \leqq x+h$, a contradiction. Thus, $a=0$ and $g<0$. 
CoROLlary. If $H$ is the intersection of all nonzero o-ideals of $G$, then every positive element of $G \backslash H$ exceeds every element of $H$.

Proof. Let $0<g \in G \backslash H$ and $h \in H$. By Lemma 3.1, $H$ is an $o-$ ideal of $G$ so there is $h^{\prime} \in H^{+}$such that $h^{\prime} \geqq h$. Now $h^{\prime}-g \in G \backslash H$ and $h^{\prime}-g<h^{\prime}$ so $h^{\prime}-g<0, h \leqq h^{\prime}<g$ and the corollary follows.

As a final observation before we turn to the main proof of this section, we note that if $G$ has no proper $o$-ideals then $G$ is a subgroup of the naturally ordered real numbers. This is a special case of 4.6 in [1].

THEOREM 3.1. A Riesz group $G$ is a pl-group if and only if $G$ satisfies.

(*) for each $g \in G$, there is $a \in G^{+}$such that $g \leqq a$ and whenever $0 \leqq x, g \leqq x$ then $a \leqq x+h$ for some $h \in \mathscr{I}^{*}(a) \cap \mathscr{I}^{*}(a-g)$.

Proof. Let $g \in G$ and $a$ satisfy (*) for $g$. We show $a$ and $a-g$ are $p$-disjoint. If $a=0$ or $a=g$, the result easily follows so we assume $g \| 0$. Let $M \in \mathscr{C}(\alpha)$ and let $M^{\prime}$ be the intersection of all $o$-ideals of $G$ that properly contain $M$. Then $M^{\prime}$ is an o-ideal of $G$, $a \in M^{\prime}, M^{\prime} / M$ is $o$-isomorphic to a subgroup of the naturally ordered real numbers and if $M<X \in(G / M) \backslash\left(M^{\prime} / M\right)$, then $X>M^{\prime} / M$.

If $(a-g)+M \geqq a+M$, then there is $m \in M^{+}$such that $a-g+$ $m \geqq a$, so $m \geqq g$. By $\left({ }^{*}\right), 0<a \leqq m+h$ where $h \in \mathscr{M}^{*}(a) \cap$ $\mathscr{L}^{*}(a-g)$.

Thus, $m+h \in M$ and $a \in M$, a contradiction. Since $(a-g)+M$ is comparable to $a+M$, we must have $(a-g)+M<a+M$, so there is $m \in M$ such that $a>(a-g)+m$. Let $m^{\prime} \in M$ such that $m^{\prime}<m$, $m^{\prime}<0$, then $g-m^{\prime}>g$ and $g-m^{\prime}>0$. Thus, by $\left(^{*}\right), a \leqq\left(g-m^{\prime}\right)+h^{\prime}$ where $h^{\prime} \in \mathscr{K}^{*}(a) \cap \mathscr{C}(a-g)$, and $0<a-g \leqq-m^{\prime}+h^{\prime} \in M$. By convexity $a-g \in M$ so $a-g \in \mathscr{l}^{*}(a)$.

By interchanging the roles of $a$ and $a-g$ in the above we are led to the conclusion that $a+M<(a-g)+M$ where $M \in \mathscr{M}(a-g)$. There then is $m \in M^{+}$such that $a<(a-g)+m$ so $g<m$. As always, $a \leqq m+h$ with $h \in \mathscr{L}^{*}(a) \cap \mathscr{l}^{*}(a-g)$ so $a \in M$. Thus, $a$ and $a-g$ are $p$-disjoint and $G$ is a $p l$-group.

The necessity follows from the remarks at the beginning of this section.

4. Pseudo-disjoint elements. Throughout this section we assume $G$ is a $p l$-group. We have shown if $g \in G$ and $g=a-b=x-y$ where $a, b$ and $x, y$ are pairs of $p$-disjoint elements then

$$
a-x=b-y \in \mathscr{C}^{*}(a) \cap \mathscr{H}^{*}(b) \text {. }
$$


However, the converse of this is not true. For if $K=R_{1}+R_{2}+R_{3}$ (the cardinal sum) where each $R_{i}$ is the real numbers, $i=1,2,3$; then $K$ is an $l$-group so, of course, a pl-group. Clearly, $(1,-1,0)=$ $(1,0,0)-(0,1,0)$ where $(1,0,0),(0,1,0)$ are $p$-disjoint. Now $(1,0,0)$ has exactly one value namely $M_{1}=R_{2}+R_{3}$ and $(0,1,0)$ has the value $M_{2}=R_{1}+R_{3}$. Thus, $R_{3}=M_{1} \cap M_{2}$ and if $0 \neq h \in R_{3}$ it is clear that $(1,0,0)+(0,0, h)=(1,0, h)$ and $(0,1,0)+(0,0, h)=(0,1, h)$ are not $p$-disjoint but $(1,-1,0)=(1,0, h)-(0,1, h)$.

We now show how pairs of $p$-disjoint elements $a, b$ and $x, y$ are related, when $g=a-b=x-y$. Assume $a$ and $b$ are $p$-disjoint and let $K=\{0 \leqq m \in G \mid m \leqq a, m \leqq b\}$. Clearly, $K$ is convex. If $m_{1}, m_{2} \in K$, then by the Riesz interpolation property, there is an element $m \in G$ such that $m_{1} \leqq m \leqq a$ and $m_{2} \leqq m \leqq b$. Moreover, $2 m \geqq m_{1}+m_{2} \geqq 0$ and by (9), $2 m \leqq a, 2 m \leqq b$ since $a$ and $b$ are $p$-disjoint. Thus, $2 m \in K$ so $m_{1}+m_{2} \in K$ and $K$ is a convex subsemigroup of $G^{+}$that contains 0 . Let $H$ be the $o$-ideal of $G$ that is generated by $K$. It is well known that $H^{+}=K$ and any $x \in H$ can be written $x=h_{1}-h_{2}$ where $h_{1}, h_{2} \in K$. Thus $H<a$ and $H<b$. We denote by $H(a, b)$ the $o$-ideal generated by $\{0 \leqq m \in G \mid m \leqq a, m \leqq b\}$ for $p$-disjoint elements $a, b$.

Lemma 4.1. If $a$ and $b$ are p-disjoint and $m \in H(a, b)$, then $\mathscr{C}(a)=\mathscr{C}(a+m)$ and $\mathscr{C l}(b)=\mathscr{H}(b+m)$.

Proof. We first consider $0 \leqq m \in H(a, b)$. Since $a \geqq a-m \geqq 0$ and $a-m \geqq a-b$ (2.1) implies $a-m$ and $b-m$ are $p$-disjoint, so $\mathscr{C l}(a)=\mathscr{C l}(a-m), \mathscr{L}(b)=\mathscr{C}(b-m)$ by $(2.2)$.

If $M \in \mathscr{C}(a+m)$, then $a-m \notin M$ so there is $M^{\prime} \supseteqq M$ such that $M^{\prime} \in \mathscr{C l}(a-m)=\mathscr{C}(a)$. Since $0 \leqq m \leqq b \in M^{\prime}, \quad m \in M^{\prime}$ so $M=$ $M^{\prime} \in \mathscr{M}($ a). Conversely, if $M \in \mathscr{M}(a)$ then $0 \leqq m \leqq b \in M$ implies $m \in M$ so $a+m \notin M$ and $M \in \mathscr{C}(a+m)$. Hence, $\mathscr{M}(a)=\mathscr{M}(a+m)$. Similarly, $\mathscr{C}(b)=\mathscr{M}(b+m)$.

For an arbitrary element $m \in H(a, b)$ there are elements $m_{1}$, $m_{2} \in H(a, b)$ such that $m_{1} \leqq 0$ and $m_{1} \leqq m, 0 \leqq m_{2} \leqq a, m \leqq m_{2} \leqq b$. Hence, $0 \leqq a+m_{1} \leqq a+m$ and $0 \leqq a+m \leqq a+m_{2}$. By the above, $\mathscr{M}(a)=\mathscr{M}\left(a+m_{1}\right)=\mathscr{M}\left(a+m_{2}\right)$. If $M \in \mathscr{C}(a+m)$, then $a+m_{2} \notin M$ so $M \in \mathscr{C}\left(a+m_{2}\right)=\mathscr{C}(a)$. Conversely, if $M \in \mathscr{C}(a)$, then $m \in M$ and $M \in \mathscr{C}\left(a+m_{1}\right)$ so $a+m \notin M$ and $M \in \mathscr{C}(a+m)$. Thus, for any $m \in H(a, b), \mathscr{M}(a)=\mathscr{M}(a+m)$. In a similar manner $\mathscr{C}(b)=$ $\mathscr{L}(b+m)$.

We note at this point that if $0 \leqq m \in H(a, b)$, then $0 \leqq m \leqq a$ implies $m \in \mathscr{M}^{*}(b)$ and $0 \leqq m \leqq b$ implies $m \in \mathscr{M}^{*}(a)$. Consequently, $H(a, b) \subset \mathscr{H}^{*}(a) \cap \mathscr{L}^{*}(b)$.

LemmA 4.2. If $a$ and $b$ are p-disjoint in $G$, then $a+m$ and 
$b+m$ are $p$-disjoint if and only if $m \in H(a, b)$.

Proof. Let $a$ and $b$ be $p$-disjoint and $m \in H(a, b)$, since $\mathscr{M}(a)=$ $\mathscr{M}(a+m), \quad b, m$ and hence $b+m \in \mathscr{M}^{*}(a+m)$. Dually, $a+$ $m \in \mathscr{K}^{*}(b+m)$ so $a+m$ and $b+m$ are $p$-disjoint.

Conversely, if $a+m$ and $b+m$ are $p$-disjoint, then $a \geqq-m$, $b \geqq-m$ so there is $h \in G$ such that $a \geqq h \geqq 0$ and $b \geqq h \geqq-m$. This implies $h \in H(a, b)$. Since $\mathscr{M}(a)=\mathscr{M}(a+m)$ and $\mathscr{M}(b)=$ $\mathscr{M}(b+m)$ we have $m \in \mathscr{M}^{*}(a) \cap \mathscr{M}^{*}(b)$. Now if $M \in \mathscr{M}(a-m)$ and $a+m \in M$, then $a \notin M$, so $M \in \mathscr{C}(a)=\mathscr{M}(a+m)$ and $a+m \notin M$, a contradiction. Thus, $a+m \notin M$ so $M \in \mathscr{M}(a+m)=\mathscr{M}(a), a \notin M$, $b \in M$. Therefore $M<a+M=(a-m)+M$. By (8), $a-m>0$. A similar argument shows $b>m$. Finally, by the Riesz interpolation property, there is an element $h^{\prime} \in G$ such that $a \geqq h^{\prime} \geqq 0$ and $b \geqq h^{\prime} \geqq m$. Thus, $h^{\prime} \in H(a, b)$ and we have $h^{\prime} \geqq m \geqq-h$ so $m \in H(a, b)$.

COROLLARY. If $a$ and $b$ are $p$-disjoint in $G$, then $a \wedge b=0$ if and only if $H(a, b)=0$.

As a consequence of Lemma 4.2 we can associate with $g=a-b$, $a$ and $b$ p-disjoint, the o-ideal $H(a, b)$. Moreover, $H(a, b)$ depends only on $g$ and is independent of the representation of $g$ as the difference of $p$-disjoint elements. To show this, let $g=x-y$ where $x$ and $y$ are also $p$-disjoint. Then by (2.2) $\mathscr{M}(a)=\mathscr{M}(x)$ and $\mathscr{M}(b)=\mathscr{M}(y)$. If $0 \leqq k \in H(x, y)$ then $k \in \mathscr{M}^{*}(a) \cap \mathscr{M}^{*}(b)$ and $a+k, b+k$ are $p$ disjoint so $k \in H(a, b)$ and $H(x, y) \subseteq H(a, b)$. Dually, we can show $H(a, b) \subseteq H(x, y)$ so $H(a, b)=H(x, y)$.

Using the above we can easily show a pl-group $G$ satisfies

$\left({ }^{*}\right)$ for each $g \in G$, there is $a \in G^{+}$such that $g \leqq a$ and whenever $0 \leqq x$, and $g \leqq x$, then $a \leqq x+h$ for some $h \in H(a, a-g)$.

To see this, let $g \in G$ and $a$ satisfy $\left({ }^{*}\right)$ for $g$. If $0 \leqq x, g \leqq x$ there is $z \in G$ such that $a \geqq z \geqq 0$ and $x \geqq z \geqq g$ since every $p l$-group is a Riesz group. By (2.1), $z$ and $z-g$ are $p$-disjoint and since $a=$ $z+(a-z)$ and $a-g=(z-g)+(a-z)$ we have $a-z \in H(z, z-g)=$ $H(a, a-g)$. Therefore, $x \geqq z=a-(a-z)$ so $x+(a-z) \geqq a$.

We have shown, that in a pl-group $G, H(a, b)$ is the o-ideal generated by $K=\{0 \leqq m \in G \mid m \leqq a, m \leqq b\}$ for $a$ and $b p$-disjoint, and $H(a, b)^{+}=K$. If we now let $H(x, y)$ be the $o$-ideal generated by $K=\{0 \leqq m \in G \mid m \leqq x, m \leqq y\}$ for arbitrary positive elements $x$ and $y$, it may happen that $H(x, y)^{+} \neq K$ and the following example shows $\left({ }^{* *}\right)$ is not sufficient for a Riesz group $G$ to be a pl-group.

Let $R$ be the naturally ordered real numbers and $G=R+R$. Let $(u, v) \in G$ be positive if $v>0$ or $v=0$ and $u=0$. Then $G$ is a Riesz group but $G$ is not a pl-group. If $g=\left(g_{1}, g_{2}\right) \in G$ and $g_{2}>0$ 
let $a=g$; if $g_{2}<0$ let $a=0$. In either case $H(a, a-g)=0$ and $a$ satisfies $\left({ }^{*}\right)$ for $g$. If $g_{2}=0$ and $g_{1}=0$ take $a=0$. If $g_{2}=0$ and $g_{1} \neq 0$ let $a=\left(a_{1}, a_{2}\right)$ where $a_{2}>0$. Then $a>0, a>g$ and $H(a, a-g)=G$. For any $b=\left(b_{1}, b_{2}\right) \geqq(0,0)$ and $\left(b_{1}, b_{2}\right) \geqq\left(g_{1}, g_{2}\right)$ we must have $b_{2}>0$. If $h=\left(0, a_{2}\right)$, then $\left(a_{1}, a_{2}\right)<\left(b_{1}, b_{2}\right)+\left(0, a_{2}\right)$ and $h \in H(a, a-g)$. Thus $\left({ }^{* *}\right)$ holds.

\section{REFERENCES}

1. P. Conrad, Representations of partially ordered abelian groups as groups of realvalued functions, Acta. Math. 116 (1966), 199-221.

2. J. R. Teller, On partially ordered groups satisfying the Riesz interpolation property, Proc. Amer. Math. Soc. 16 (1965), 1392-1400.

Received July 3, 1967.

GEORGETOWN UNIVERSITY

WAShington, D. C. 



\section{PACIFIC JOURNAL OF MATHEMATICS}

\section{EDITORS}

\section{H. ROYDEN}

Stanford University

Stanford, California

\author{
R. R. Phelps \\ University of Washington \\ Seattle, Washington 98105
}

\section{J. DugundJI}

Department of Mathematics University of Southern California Los Angeles, California 90007

\section{RICHARD ARENS}

University of California

Los Angeles, California 90024

\section{ASSOCIATE EDITORS}

\section{E. F. BECKENBACH}

B. H. NEUMANN

F. WOLF

K. YOSIDA

\section{SUPPORTING INSTITUTIONS}

UNIVERSITY OF BRITISH COLUMBIA CALIFORNIA INSTITUTE OF TECHNOLOGY UNIVERSITY OF CALIFORNIA MONTANA STATE UNIVERSITY UNIVERSITY OF NEVADA NEW MEXICO STATE UNIVERSITY OREGON STATE UNIVERSITY UNIVERSITY OF OREGON OSAKA UNIVERSITY UNIVERSITY OF SOUTHERN CALIFORNIA

\author{
STANFORD UNIVERSITY \\ UNIVERSITY OF TOKYO \\ UNIVERSITY OF UTAH \\ WASHINGTON STATE UNIVERSITY \\ UNIVERSITY OF WASHINGTON \\ AMERICAN MATHEMATICAL SOCIETY \\ CHEVRON RESEARCH CORPORATION \\ TRW SYSTEMS
}

NAVAL WEAPONS CENTER

Mathematical papers intended for publication in the Pacific Journal of Mathematics should be in typed form or offset-reproduced, double spaced with large margins. Underline Greek letters in red, German in green, and script in blue. The first paragraph or two must be capable of being used separately as a synopsis of the entire paper. It should not contain references to the bibliography. Manuscripts, in duplicate if possible, may be sent to any one of the four editors. All other communications to the editors should be addressed to the managing editor, Richard Arens, University of California, Los Angeles, California 90024.

Each author of each article receives 50 reprints free of charge; additional copies may be obtained at cost in multiples of 50 .

The Pacific Journal of Mathematics is published monthly. Effective with Volume 16 the price per volume (3 numbers) is $\$ 8.00$; single issues, $\$ 3.00$. Special price for current issues to individual faculty members of supporting institutions and to individual members of the American Mathematical Society: $\$ 4.00$ per volume; single issues $\$ 1.50$. Back numbers are available.

Subscriptions, orders for back numbers, and changes of address should be sent to Pacific Journal of Mathematics, 103 Highland Boulevard, Berkeley 8, California.

Printed at Kokusai Bunken Insatsusha (International Academic Printing Co., Ltd.), 7-17, Fujimi 2-chome, Chiyoda-ku, Tokyo, Japan.

PUBLISHED BY PACIFIC JOURNAL OF MATHEMATICS, A NON-PROFIT CORPORATION

The Supporting Institutions listed above contribute to the cost of publication of this Journal, but they are not owners of publishers and have no responsibility for its content or policies. 


\section{Pacific Journal of Mathematics}

\section{Vol. 27, No. $2 \quad$ February, 1968}

Leonard E. Baum and George Roger Sell, Growth transformations for

functions on manifolds ............................ 211

Henry Gilbert Bray, A note on CLT groups ................... 229

Paul Robert Chernoff, Richard Anthony Rasala and William Charles

Waterhouse, The Stone-Weierstrass theorem for valuable fields....... 233

Douglas Napier Clark, On matrices associated with generalized

interpolation problems ................................

Richard Brian Darst and Euline Irwin Green, On a Radon-Nikodym theorem for finitely additive set functions . ...................... 255

Carl Louis DeVito, A note on Eberlein's theorem..................... 261

P. H. Doyle, III and John Gilbert Hocking, Proving that wild cells exist . . . 265

Leslie C. Glaser, Uncountably many almost polyhedral wild $(k-2)$-cells in

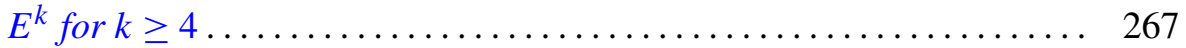

Samuel Irving Goldberg, Totally geodesic hypersurfaces of Kaehler

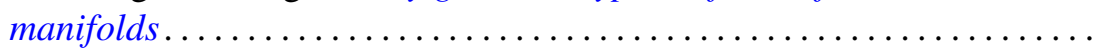

Donald Goldsmith, On the multiplicative properties of arithmetic functions .................................... 283

Jack D. Gray, Local analytic extensions of the resolvent ............ 305

Eugene Carlyle Johnsen, David Lewis Outcalt and Adil Mohamed Yaqub,

Commutativity theorems for nonassociative rings with a finite division ring homomorphic image ....................

André (Piotrowsky) De Korvin, Normal expectations in von Neumann

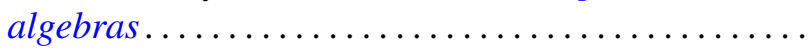

James Donald Kuelbs, A linear transformation theorem for analytic

Feynman integrals..........................

W. Kuich, Quasi-block-stochastic matrices ................... 353

Richard G. Levin, On commutative, nonpotent archimedean

semigroups ............................... 365

James R. McLaughlin, Functions represented by Rademacher series ... . . . 373

Calvin R. Putnam, Singular integrals and positive kernels............ 379

Harold G. Rutherford, II, Characterizing primes in some noncommutative

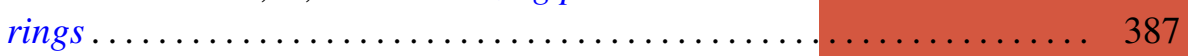

Benjamin L. Schwartz, On interchange graphs................... 393

Satish Shirali, On the Jordan structure of complex Banach *algebras . . . . . 397

Earl J. Taft, A counter-example to a fixed point conjecture............. 405

J. Roger Teller, On abelian pseudo lattice ordered groups ..... 